Estrategia Comunicacional en la Gestión Institucional del Consejo Social de la Universidad Nacional de La Plata (UNLP)

Verónica Veira

Revista ES (en y sobre Educación Superior)

Vol.1, N¹-2 / Fecha de publicación: 27/12/2021

e-ISSN: 2718-6539

https://revistas.unlp.edu.ar/ES/index

IIES - Facultad de Odontología

DOI: https://doi.org/10.24215/27186539e042

\title{
Estrategia Comunicacional en la Gestión Institucional del Consejo Social de la Universidad Nacional de La Plata (UNLP)
}

\author{
Communication Strategy in Institutional Management of the Social \\ Council of the National University of La Plata (UNLP)
}

Estratégia Comunicacional em Gestão Institucional do Conselho Social da Universidade Nacional de La Plata (UNLP)

Verónica Veira

Asistente en la Prosecretaria de Extensión -UNLP veronicaveira@gmail.com

\section{Resumen}

El presente resumen del trabajo final integrador (TFI), en el marco de la Especialización en Gestión de la Educación Superior (EGES), contempla en 
principio un análisis y estudio en torno a la historia, alcances y perspectivas del Consejo Social (CS) de la Universidad Nacional de La Plata. El objetivo es proponer un modelo de gestión de procesos y herramientas de comunicación interna y externa, a partir de mi rol como Directora de Gestión Institucional del Consejo Social de la UNLP, asegurando el funcionamiento del Plenario y de las Comisiones de Trabajo del CS; gestionando los espacios y recursos del CS, a partir del seguimiento del trabajo y de la agenda de los diferentes espacios del CS. Asimismo, asistiendo a la Dirección General del CS en sus funciones, y articulando con los procesos de gestión del resto de las Direcciones del CS. Siendo el CS una de las primeras experiencias donde la Universidad coexiste e integra gran parte de los actores sociales. Se presenta como una instancia articuladora, una mirada en la cual abordar las prácticas desde la interdisciplinariedad, y en ese sentido, profundizar la comprensión de la realidad que se integra a las prácticas y se deja atravesar por los sentidos que allí se generan. Inmersos en la dinámica cotidiana en donde los distintos espacios conforman redes de comunicación que se instituyen como prácticas socio-institucionales.

\section{Abstract}

This summary of the final integrative work (TFI), within the framework of the Specialization in Higher Education Management (EGES), initially contemplates an analysis and study around the history, scope and perspectives of the Social Council (CS) of the National University of La Plata. The objective is to propose a process management model and internal and external communication tools, based on my role as Director of Institutional Management of the Social Council of the UNLP, ensuring the functioning of the Plenary and the Working 
Committees of the SC; managing the spaces and resources of the SC, based on the monitoring of the work and the agenda of the different spaces of the SC. Likewise, assisting the General Directorate of the SC in its functions, and coordinating with the management processes of the rest of the SC Directorates. Being the CS one of the first experiences where the University coexists and integrates a large part of the social actors. It is presented as an articulating instance, a look in which to approach the practices from an interdisciplinary perspective, and in that sense, to deepen the understanding of the reality that is integrated into the practices and is allowed to pass through the senses that are generated there. Immersed in the daily dynamics where the different spaces make up communication networks that are instituted as socio-institutional practices.

\section{Resumo}

Esta síntese do trabalho integrativo final (TFI), no âmbito da Especialização em Gestão do Ensino Superior (EGES), contempla inicialmente uma análise e um estudo em torno da história, âmbito e perspectivas do Conselho Social (CS) da Universidade Nacional de La Plata. O objetivo é propor um modelo de gestão de processos e ferramentas de comunicação interna e externa, com base na minha função de Diretor de Gestão Institucional do Conselho Social da UNLP, garantindo o funcionamento do Plenário e das Comissões de Trabalho do SC; gestão dos espaços e recursos do SC, com base no acompanhamento dos trabalhos e da agenda dos diferentes espaços do SC. Da mesma forma, coadjuvar a Direção-Geral do CS em suas funções e coordenar os processos de gestão das demais Diretorias do CS. Sendo o CS uma das primeiras experiências onde a Universidade convive e integra grande parte dos atores 
sociais. Apresenta-se como uma instância articuladora, um olhar no qual abordar as práticas a partir de uma interdisciplinaridade e, nesse sentido, aprofundar a compreensão da realidade que se integra às práticas e se deixa passar pelos sentidos aí gerados. Imerso nas dinâmicas quotidianas onde os diferentes espaços constituem redes de comunicação que se instituem como práticas socioinstitucionais.

\section{Palabras clave}

Comunicación, Gestión, Estrategia, Participación, Universidad

\section{Keywords}

Communication, Management, Strategy, Participation, University

\section{Palavras chave}

Comunicação, Gestão, Estratégia, Participação, Universidade

\section{La Universidad Nacional de La Plata}

La UNLP es esencialmente pública, autónoma, cogobernada y reformista. Los primeros días de 2018 están redondeando un siglo de la gesta de la Reforma Universitaria de 1918, que modeló el ideario y el paradigma aspirado para nuestra Institución. Avivan reflexiones sobre la vigencia de esos postulados, las imposiciones de nuestro tiempo y la necesidad de proyectar esta institución en el futuro inmediato y mediato comprendiendo el momento actual de nuestra historia, el escenario deseado sobre el cual queremos proyectar su futuro y las necesarias prioridades que permiten consolidar el proceso permanente de transformación positiva de la universidad argentina, moderna y vigente en sus 
aspiraciones, pero asentada en cimientos sólidos, construidos con principios irrenunciables e imprescriptibles. ${ }^{1}$

La universidad debe ser hoy un espacio para tener ideas y discutirlas, alejada del pensamiento único, promotora del pensamiento diverso, original y propio; caracterizada por estar llena de inquietudes, rechazos, audacias, escrúpulos y esperanzas que custodian el comportamiento ético, transparente, republicano y democrático de sus miembros. Esto no significa resignar calidad (ni pertinencia, ni la siempre bien defendida búsqueda de la excelencia), por el contrario, significa agregarle responsabilidades al Sistema.

Ese es el verdadero contrato que se firmó cuando este modelo fue concebido para desarrollar al país y multiplicar las oportunidades para el progreso colectivo. La calidad y la masividad, asociada al esfuerzo y al compromiso son un conjunto indisoluble. Si existe uno de estos factores, necesariamente existen los otros en la universidad pública argentina. Una institución educativa de gran complejidad como la Universidad Nacional de La Plata afronta y desarrolla centenares de programas y proyectos emergentes de políticas académicas, científicas y sociales que trazan objetivos acordes con las definiciones expuestas. Por ejemplo, el Plan Estratégico de la UNLP, es un instrumento de gestión participativa emergente de un proyecto institucional. "Pensar la Universidad es imaginarla en el mundo, en el país y en su región, aportando a sus desarrollos, enseñando, produciendo, transfiriendo y compartiendo conocimientos e integrándose desde la sociedad que la compone y de la que forma parte y se nutre, contribuyendo con su pensamiento crítico y propositivo, capaz de incidir mediante la formulación de respuestas

\footnotetext{
${ }^{1}$ Fernando Tauber: Pensar la Universidad - Lineamientos Generales. UNLP 20182022
} 
alternativas, en los cambios sociales necesarios. Se piensa a la Universidad con un rol protagónico con responsabilidad social."2

La Planificación Estratégica Situacional fue propuesta por el autor latinoamericano Carlos Matus ${ }^{3}$. El autor, en su búsqueda de teoría y metodología para la planificación y el abordaje de situaciones, evidenció la necesidad de vincular dos procesos que en general se encuentran divididos: la planificación y la gestión. La concepción de planificación estratégica planteada por Matus ${ }^{4}$ (Huertas, 1993) en su propuesta, entiende a un sujeto que se encuentra comprendido en el "objeto planificado", reconociendo que existe más de una explicación verdadera producto de la perspectiva que cada actor social le imprime a la situación analizada.

El pensamiento estratégico establece la necesaria vinculación entre la situación analizada y la formulación de objetivos conscientemente seleccionados para la modificación de los principales problemas identificados, en función de decisiones que se toman a partir de un análisis de viabilidad de la acción propuesta. La categoría que integra la propuesta de Gestión Estratégica Planificada es la de organización. La hemos incorporado ya que la complejidad y la necesidad de comprender lo que ocurre, la forma de estructurarse, permanecer y modificarse resulta vital para sostener los procesos de gestión planificada.

\footnotetext{
${ }^{2}$ Fernando Tauber: Pensar la Universidad - Proyecto Institucional UNLP 2018-2022

${ }^{3}$ Carlos Matus fue un economista chileno y Ministro de Economía, Fomento y Reconstrucción de Chile durante el gobierno de Salvador Allende.

${ }^{4}$ (Huertas, 1993) Huertas, F. (Marzo de 1993). El Método PES, Planificación Estratégica Situacional. Obtenido de:

https://www.cepal.org/ilpes/noticias/paginas/2/36342/LIBRO_ENTREVISTA_CON_MA TUS.pdf
} 
Por lo tanto, la propuesta es sistematizar un conjunto de dimensiones de análisis y de operaciones incluidas en cada una de ellas, que consideramos pueden ser tenidas en cuenta en forma total o parcial cuando se aborda un proceso de gestión en una organización que pretende ser estratégico y planificado.

Intervenir desde el enfoque de la Gestión Estratégica Planificada es proponer desde la situación, no desde fuera, es entender a las organizaciones como espacios donde se desarrollan procesos de articulación de recursos y toma de decisiones signados por la incertidumbre y la crisis, instancias en donde actores con intereses diversos pugnan por llevar adelante perspectivas y acciones.

Conocerla implica, por lo tanto, una fase de (re)conocimiento y aprendizaje que devele los modos de trabajo, las costumbres, la cultura organizacional y también los diferentes grupos, la existencia de coincidencias y disidencias sobre los temas que atraviesan y significan a la organización entre otros componentes de análisis que ayuden a comprender el escenario.

Planear es obtener una visión global del terreno; es hacer un plano de las diferentes relaciones y sus variables, combinar los procesos y los acontecimientos para la construcción de escenarios difíciles de prever.

"La prospectiva lleva a establecer escenarios que son descripciones de una situación futura, y también marcan esos caminos necesarios para que esa situación se concrete, o se construya en sí misma. Sin embargo, un escenario no es un fin: es un paso previo para la toma de decisiones, para la acción. Es un paso para el planeamiento, aunque no siempre es anterior. Esta relación secuencial, evolutiva, no siempre es la mejor ni es la posible. Muchas veces se 
superponen instancias de planeamiento conjuntamente con la construcción de escenarios y se produce un efecto de vaivén." 5(Giordano, 2009, pág. 86).

\section{El Consejo Social de la Universidad Nacional de La Plata}

El espacio se creó en septiembre de 2010 y se constituye en un ámbito de reflexión dirigido a planificar y fortalecer el desarrollo social, y el desarrollo de los debates que aportan a transformar políticas públicas y a la propia UNLP. Alí se acuerdan en conjunto mecanismos de acción en el ámbito social y productivo, tendientes a mejorar las condiciones de vida de la población.

Para llevar adelante todas estas acciones la comunicación interna y externa tiene un rol muy importante dentro del CS. En este sentido, se destacan las principales fortalezas del Consejo que lo caracterizan en el cual los sectores que lo integran se encuentran fuertemente comprometidos. Existe un muy importante apoyo de Facultades y Colegios a las iniciativas del CS. Además, perciben que pueden encauzar problemáticas y buscar soluciones dentro de este ámbito. Permitiendo abrir nuevas líneas de trabajo dentro de la UNLP a partir del camino recorrido.

La experiencia hasta el momento se ha mostrado muy valiosa hacia el interior de la UNLP, y también en relación a muchos temas de la agenda social de la región. El compromiso de los integrantes del Consejo y la capacidad de articulación con otros espacios dentro y fuera de la UNLP presentan grandes potencialidades.

Funcionamiento del Consejo Social (CS)

\footnotetext{
${ }^{5}$ Giordano, C. (Junio de 2009). 23 Tesis sobre la tesis - La comunicación para la transformación de la gestión educativa. Obtenido de Capitulo 8:

http://sedici.unlp.edu.ar/bitstream/handle/10915/35341/Documento completo.pdf?sequ ence $=3 \&$ is Allowed $=y$
} 
La estructura interna del CS fue tomando forma a lo largo de estos diez años, y es por esto que, en el año 2018 se decidió ampliar su estructura dándole lugar a seis direcciones para distribuir las principales tareas. La estructura está compuesta por la Dirección General del Consejo Social, la Dirección de Gestión Institucional, la Dirección de Gestión Social, la Dirección de articulación con Educación, la Dirección de articulación con Innovación y Transferencia, la Dirección de articulación con Extensión y Relaciones Institucionales.

Dinámica participativa

El CS se estructura a partir de un espacio Plenario, donde sus integrantes hacen uso de la palabra, aportan su mirada y experiencia en la totalidad de los temas de agenda, y en comisiones de trabajo.

La agenda de trabajo del Consejo ha ido creciendo a lo largo de estos diez años, y actualmente cuenta con reuniones plenarias mensuales y seis Comisiones permanentes de trabajo que abordan temas diversos como:

1. Tierra, Vivienda y Hábitat,

2. Economía Popular, Social y Solidaria

3. Salud,

4. Niñez, Adolescencia y Juventud,

5. Educación,

6. Derechos Migrantes

Además, también funciona en el marco del CS

- el Programa de Elaboración de Alimentos a nivel local (PESAL),

- el Programa de Promoción y Fortalecimiento de la Agricultura Familiar,

- la subcomisión de Residuos Sólidos Urbanos,

- del Relevamiento sobre los Sitios de Distribución de Alimentos,

- la mesa técnica del sobre uso terapéutico de Cannabis, 
- la mesa técnica de Salud Mental.

Las Diplomaturas en:

- Gestión del Hábitat y Promoción Integral del Territorio,

- Promoción y Gestión de la Economía Popular, Social y Solidaria,

- Promoción de Derechos de Infancias y Juventudes,

- Promoción de la Seguridad Humana de los Habitantes,

- Promoción y Gestión de la Salud Comunitaria.

El plenario del Consejo Social está compuesto por dependencias del Gobierno de la Provincia de Buenos Aires, representantes de las cámaras legislativas, los municipios de la Región Capital, centrales sindicales, organizaciones territoriales, cooperativas, organismos de ciencia y tecnología y diferentes actores de la comunidad universitaria.

Es conducido por el presidente de la UNLP, y por la Dirección General del Consejo Social de la UNLP.

Organización en Comisiones

La organización en comisiones surge del desarrollo de un diagnóstico en común en la reunión plenaria, que agruparon diferentes problemáticas.

Se fueron dividiendo en comisiones propuestas sobre ejes temáticos, mayormente aportados por el interés de los movimientos sociales con la ayuda e intervención de la UNLP, en el cual se fueron afianzando e integrando con el transcurso de los años.

La comunicación en la organización en comisiones, sirve para proporcionar la integración y generar ambientes de trabajo donde sea posible conjugar los interese sectoriales y los colectivos, orientados a mejorar integralmente tanto la calidad de vida del conjunto de la población, como la formación universitaria, 
con el aporte de todos los sectores sociales, aportando además la visión de la UNLP en temas centrales en el debate de la agenda social en la actualidad.

Organizar y comunicar requiere de un plan estratégico que le dé sentido y permita fundamentar y armonizar las necesarias y complejas relaciones interculturales o interpersonales que ya existen en una organización con una necesidad de cambio organizacional o comunicacional fundada en un análisis y evaluación de lo disponible para medir aquellos puntos fuertes que, en todo caso, conviene reforzar y los puntos negativos o débiles necesarios de transformar.

No obstante, para que la comunicación esté organizada, debe poder caracterizarse.

“...tener una finalidad, es decir, debe estar vinculada a objetivos y a un plan de conjunto; debe ser multidireccional, es decir, de arriba hacia abajo, de abajo hacia arriba, transversal, interna-externa, etcétera; debe estar instrumentada y valerse de herramientas, soportes, dispositivos, indicadores seleccionados en función de los objetivos; debe estar adaptada integrando sistemas de información administrables, administrados y adaptados a las necesidades específicas de cada sector teniendo siempre en cuenta la cultura del medio; debe ser flexible, para integrar lo informal y crear estructuras que lo favorezcan". (Tauber $)^{6}$

\footnotetext{
${ }^{6}$ Tauber, F. (s.f.). La planificación, la gestión, la comunicación institucional. Consideraciones en torno al estado de la cuestión referido al objeto de estudio y su contexto.

http://sedici.un/p.edu.ar/bitstream/handle/10915/1965/l. La planificaci\%C3\%B3n la gesti\%C3\%B3n la comunicaci\%C3\%B3n institucional. Consideraciones en torno al estado de la cuesti\%C3\%B3n referido al objeto de estudio y su contexto.pdf? sequence $=3 \&$ isAllowe
} 


\section{Estrategia Comunicacional de la Dirección de Gestión Institucional}

La Dirección de Gestión Institucional del Consejo Social de la UNLP, tiene como labor principal asegurar el funcionamiento del Plenario y de las Comisiones de Trabajo del CS; gestionando los espacios y recursos del CS, a partir del seguimiento del trabajo y de la agenda de los diferentes espacios del CS. Entendiendo la comunicación institucional como todos los procesos de producción y recepción de mensajes que se realizan en una organización tanto en su interior y también hacia el exterior que permita optimizar la gestión del CS como organización fortalecimiento la identidad.

Es necesario tener una comunicación interna sólida y bien organizada, para que la organización se proyecte y comunique de forma eficaz a sus integrantes. En ese marco las acciones de comunicación de la UNLP se mueven en un complejo escenario donde se ponen en juego nuevas habilidades de producción para soportes tecnológicos en constante evolución y complejidad, y las variables políticas que una institución universitaria pública presenta. En el cual el CS presenta una estructura singular que no tiene equivalente en ninguna de los de las Universidades del país.

El CS planifica desde una perspectiva estratégica estableciendo las principales orientaciones y prioridades adoptando las decisiones básicas sobre su desarrollo a corto, mediano y largo plazo teniendo en cuenta la relación con su contexto, evaluaciones y presupuestos.

Provee una planificación por objetivos de conocimiento a lograr con los distintos actores, que resulta a la vez: previsora, racional, flexible y promotora de cambios a corto, mediano y largo plazo. Es una tarea permanente en el plenario del CS. En el cual se planifica la acción para luego ser comunicada. Especifica metas de producto que dan cuenta de la gestión de la innovación en 
los distintos niveles: acciones, programas, proyectos y áreas estratégicas y permite la autoevaluación.

Para abordar el problema, se propone el diagnóstico y diseño de una estrategia comunicacional, donde la comunicación se entiende como momento vinculante de la diversidad sociocultural, convocando a los diversos actores vinculados con las publicaciones, para lograr una transformación o cambio social a partir del encuentro conversacional.

En este sentido se utilizan todos los canales de difusión en la estrategia de comunicación interna y externa. Articulando con otras áreas de comunicación dentro y fuera de la UNLP. Conformando equipos multidisciplinarios capaces de instalar conversaciones múltiples en lugares específicos o miradas complejas cuando no es posible disponer de equipos.

Este caso hace referencia a las comisiones de trabajo del CS. Desde la comunicación, que intenta dar una mirada propia de la realidad, se produce una construcción colectiva del conocimiento. Los actores sociales se empoderan del conocimiento, y desde sus propios saberes colectivos transforman su realidad desde el presente.

Por último, reconocer intereses y necesidades de los actores. Una estrategia de comunicación es un proyecto de comprensión que asume a la comunicación como espacio de encuentro de los actores.

Gestión y estrategias de la Comunicación Interna

En la comunicación interna, es decir, la comunicación entre los miembros del CS, es importante tener en cuenta qué queremos decir y cómo queremos decirlo, y de qué manera se transmite mejor el mensaje sabiendo que existe una diferencia entre lo que se recibe y lo que se percibe, por lo que se hace de 
vital importancia fomentar la participación y el compromiso de las personas que lo integran.

A través de la comunicación interna se facilita el despliegue de todos los instrumentos comunicacionales con el objetivo de:

- Promover la comunicación entre los miembros

- Reducir los focos de conflicto interno a partir del fortalecimiento de la cohesión de los miembros

- Contribuir a la creación de espacios de información, participación y opinión

Para llevar a cabo estos objetivos se tienen en cuenta:

- realizar las convocatorias a los plenarios mensuales y a las comisiones de trabajo.

- sistematización de cada convocatoria y su posterior envío

- registro de todos los integrantes

Las convocatorias para garantizar la representación de todos los espacios que participan en el plenario se realiza vía correo electrónico. Se detalla el día, horario y lugar. Son convocadas con diez días de anticipación. Se envía un recordatorio electrónico cinco días antes, en el que se detalla el temario propuesto desde la coordinación del Consejo. Se solicita confirmar asistencia, también por mail. De acuerdo a las confirmaciones, aquellos que no contestaron se chequea vía llamado telefónico o por WhatsApp. En el caso que el consejero o consejera no pueda participar se solicita el envío de un representante. Por último, se realiza la relatoría de la reunión correspondiente. Se envía la relatoría del plenario a todos los integrantes.

Los procedimientos de las convocatorias en las comisiones son mediante correo electrónico y son abiertas para todas/os los que deseen participar, 
entendiendo la importancia de los aportes y visiones de todos los sectores frente a las demandas y la agenda propuesta colectivamente. Se contempla convocar con tiempo. Las reuniones de cada comisión se definen en las instancias plenarias y siguen funcionando independientemente, así lo requieran. Finalizada la reunión se realiza una relatoría, escrita por un integrante designado previamente o por la Directora General del CS. Es revisado por el equipo central antes del envío a todos los participantes. Hay un formato preestablecido: Se menciona a los participantes, se relata la agenda de trabajo, los consensos alcanzados y las acciones que se llevarán adelante.

\section{Gestión y estrategias de la Comunicación Externa}

Respecto a la comunicación externa, la importancia reside en proyectar, promover y reforzar la imagen del CS, publicitar nuestros proyectos 0 actividades, saber a quienes nos dirigimos y conseguir la participación e interacción de nuestros participantes, o lo que es lo mismo, dar a conocer nuestra organización a los demás.

Es importante tener en cuenta cómo nos perciben y cómo queremos que nos perciban y elaborar un plan de acción de comunicación externa. En la gestión de la comunicación externa es parte de una estrategia comunicacional definida por la Dirección General de Comunicación Institucional de la UNLP, la información se difunde en las redes sociales y el portal web de la UNLP, se busca la difusión masiva de las diversas actividades y presentaciones que se desarrollan en el marco del CS.

Para esto se tiene en cuenta los siguientes puntos: 
¿Qué comunicar? Es importante comunicar contenidos basados en la estrategia formulada y los objetivos comunicacionales perseguidos, elaborando contenido en cuenta a los públicos objetivo.

¿A quiénes comunicar? En la selección de contenidos y temáticas para compartir en los espacios del CS, es necesario definir el público objetivo, es decir, al destinatario ideal. Se define cuales tienen alcance a la comunidad (charla, presentación de un proyecto, dictado de una diplomatura) y/o dentro del Consejo (reunión plenaria, una determinada comisión, mesa técnica).

¿Dónde comunicar? Es necesario especificar en qué o cuáles plataformas se comunicará (mail, WhatsApp, Facebook, Twitter, Instagram, Portal Web) dónde se va a centrar el plan de acción.

¿Cómo comunicar? Diferenciar las tácticas de cada plataforma en particular y adaptar los mensajes a cada una de ellas las cuales responden a lógicas distintas. Por ejemplo las alternativas son las redes sociales del Consejo Social (Facebook: $\quad$ https://www.facebook.com/consejosocial.unlp/, Twitter: @consejosociallp, Instagram: @ConsejoSocialUNLP)

En ellas se publican invitaciones de las actividades que surgen de la organización del plenario, comisión de trabajo o mesa técnica e información relevante para la comunidad.

En el portal web de la UNLP: https://unlp.edu.ar/consejo social, se centraliza toda la información institucional, se suben todos los resúmenes de las reuniones plenarias, hay información sobre todos los integrantes de las comisiones y también todo lo que se difunde en las redes sociales y las gacetillas de prensa de los medios locales y zonales.

En función al diagnóstico de su estructura, funcionamiento y el gran número de integrantes se han detectado ciertas debilidades comunicacionales: 
- Dificultades en la circulación de la información: la información operativa carece de una dinámica definida que garantice llegar en tiempo y forma, generando dificultades en las operaciones y pérdida de productividad.

- Reserva inadecuada de la información: no existe espacio para almacenar el conocimiento colectivo, o una plataforma que facilite la acumulación.

- Débil registro fotográfico y de las relatorías debido a la dinámica en sus actividades.

- Ausencia de sistematización y documentación de todas las tareas que se realizan.

- Falta de actualización en el portal web de la UNLP acorde a la dinámica del CS.

- Problemas para encontrar el sitio web del CS en el portal de la UNLP.

- Inexistencia de una agenda común: que permita a todos tener acceso a las reuniones y obtener confirmaciones a las mismas.

- Inclusión digital de los integrantes del CS, no todos/as cuentan con un correo electrónico y/o WhatsApp.

En la gestión cotidiana se considera que hay inconvenientes en las comunicaciones internas y externas, aún con todas las alternativas disponibles en la diversidad de herramientas/vías de comunicación, ya explicitados para informar, y para que efectivamente la información llegue a los integrantes del CS correspondientes en tiempo y forma, de manera oportuna, resultando de ello la necesidad de revisar todo el proceso, ¿es el contenido?, o será ¿la forma en que se presenta dicho contenido?, ¿serán las vías adecuadas para comunicar?. 
Nos enfrentamos ante el desafío de encontrar los ruidos en el proceso comunicacional para poder gestionar alternativas. Asumida esta como una oportunidad para que desde la estructura interna del CS se desarrollen estrategias de comunicación interna y externa dentro su dinámica de funcionamiento.

\section{Reflexiones Finales}

El Trabajo Final Integrador (TFI) consistió en una gran experiencia para el análisis del proceso interno del CS. Poniendo en valor su desarrollo a partir de la inserción concreta y cotidiana en procesos sociales específicos que hacen parte de esta realidad como un aporte a la producción de conocimiento.

Considerando la importancia de esta instancia de formación en la Gestión de la Educación Superior y reconociendo nuestra historia para tener un plan que esté por encima de circunstanciales actores, objetivos que se mantengan a pesar del cambio de contextos políticos, sociales y económicos.

Otro aspecto importante de la EGES es que permitió que nos conozcan y que comprendan nuestras lógicas de trabajo. Además, posibilitó conocer otras áreas de gestión en la UNLP, comprendiendo mejor su funcionamiento y fortaleciendo los lazos en el trabajo cotidiano.

Luego de haber analizado los conceptos de comunicación, planificación y gestión desde la perspectiva de estudio propuesta por la EGES, aplicándolas a la Dirección de Gestión institucional del Consejo Social de la UNLP, comprendemos el valor estratégico de diseñar políticas de comunicación que garanticen el diálogo al interior del CS y con la Universidad toda.

Entendimos que para profundizar el proceso de transformación se requiere que en la toma de decisiones todas las áreas estén interconectadas entre sí. 
Promover instancias de diálogo, que habiliten la participación es la forma de avanzar colectivamente para lograr los objetivos trazados. Planificar cada una de las acciones comunicacionales porque permite abarcar la comunicación interna, la interinstitucional y la externa, definiendo en cada caso cuál es la problemática prioritaria y los actores inmersos en la institución compleja, organización de base pesada, como es la UNLP.

\section{Bibliografía}

- Camps, S. (1999). Periodismo sobre catástrofes. Buenos Aires: Paulinas.

- Castillo, D. P. (s.f.). La planificación como una experiencia decisiva.

- Giordano, C. (Junio de 2009). 23 Tesis sobre la tesis - La comunicación para la transformación de la gestión educativa. Obtenido de Capitulo 8: http://sedici.unlp.edu.ar/bitstream/handle/10915/35341/Documento complet o.pdf? sequence $=3 \&$ isAllowed $=y$

- Huertas, F. (Marzo de 1993). El Método PES, Planificación Estratégica Situacional. Obtenido: de https://www.cepal.org/ilpes/noticias/paginas/2/36342/LIBRO_ENTREVISTA_ CON_MATUS.pdf

- Massoni, S. (2007). Sección: Modelo de comunicación estratégica (Tres movimientos y siete pasos para la comunicación). En S. Massoni, Estrategias. Los desafíos de la comunicación en un mundo fluido. Rosario: Homo Sapiens.

- Porta Paula. Comunicación Medios y Poder Perelló, L. (s.f.).

- Prieto Castillo, Daniel La planificación como una experiencia decisiva.

- Tauber, F. (2014). Compromiso. Gestión 2010-2014. La Plata. Tauber, F: Pensar la Universidad - Proyecto Institucional UNLP 2018-2022. 
http://sedici.unlp.edu.ar/bitstream/handle/10915/68411/Versi\%C3\%B3n 2.p df? sequence $=2 \&$ is Allowed $=y$

- Tauber, F. (s.f.). La planificación, la gestión, la comunicación institucional. Consideraciones en torno al estado de la cuestión referido al objeto de estudio y su contexto. Obtenido de:

http://sedici.unlp.edu.ar/bitstream/handle/10915/1965/I. La planificaci\%C3 \%B3n la gesti\%C3\%B3n la comunicaci\%C3\%B3n institucional. Consi deraciones en torno al estado de la cuesti\%C3\%B3n referido al objeto de estudio y su contexto.pdf?sequence=3\&isAllowe 